\title{
ORIGINAL RESEARCH \\ Differentiation of Typical and Atypical Parkinson Syndromes by Quantitative MR Imaging
}

\author{
N.K. Focke \\ G. Helms
}

P.M. Pantel

S. Scheewe

M. Knauth

C.G. Bachmann

J. Ebentheuer

P. Dechent

W. Paulus

C. Trenkwalder
BACKGROUND AND PURPOSE: The differential diagnosis of Parkinson syndromes remains a major challenge. Quantitative MR imaging can aid in this classification, but it is unclear which of the proposed techniques is best suited for this task. We, therefore, conducted a head-to-head study with different quantitative MR imaging measurements in patients with IPS, MSA-type Parkinson, PSP, and healthy elderly controls.

MATERIALS AND METHODS: Thirty-one patients and 13 controls underwent a comprehensive quantitative MR imaging protocol including R2 ${ }^{*}$-, R2- and R1-mapping, magnetization transfer, and DTI with manual region-of-interest measurements in basal ganglia regions. Group differences were assessed with a post hoc ANOVA with a Bonferroni error correction and an ROC.

RESULTS: The best separation of MSA from IPS in patients and controls could be achieved with R2*-mapping in the PU, with an ROC AUC of $\leq 0.96$, resulting in a sensitivity of $77.8 \%$ (with a specificity $100 \%)$. MD was increased in patients with PSP compared with controls and to a lesser extent compared with those with IPS and MSA in the SN

CoNCLUSIONS: Among the applied quantitative MR imaging methods, R2*-mapping seems to have the best predictive power to separate patients with MSA from those with IPS, and DTI for identifying PSP.

ABBREVIATIONS: APS $=$ atypical Parkinson syndrome; $\mathrm{AUC}=$ area under the curve; $\mathrm{CN}=$ (head of the) caudate nucleus; FA = fractional anisotropy; FLASH = fast low-angle shot; IPS = idiopathic Parkinson syndrome; MD = mean diffusivity; MPRAGE = magnetization-prepared rapid acquisition of gradient echo; MSA = multiple systems atrophy; MTR = magnetization transfer ratio; PA = globus pallidum; $\mathrm{PSP}=$ progressive supranuclear palsy; $\mathrm{PU}=$ putamen; $\mathrm{R} 1=\mathrm{T} 1$ relaxation rate; $\mathrm{R} 2=\mathrm{T} 2$ relaxation rate; $\mathrm{R}^{*}{ }^{*}=\mathrm{T} 2^{*}$ relaxation rate; $\mathrm{ROC}=$ receiver operating characteristic analysis; $\mathrm{SN}=$ substantia nigra; STEAM = stimulated echo acquisition mode.
$\mathbf{T}_{\mathrm{n}}^{\mathrm{h}}$ he differential diagnosis of Parkinson syndromes is still a major challenge for clinicians and movement disorder specialists, especially in the early stage of the disease. These syndromes comprise IPS and APSs like MSA-type Parkinson or PSP. Cranial MR imaging can aid in this important classification of patients, and several radiologic signs have already been described differentiating the atypical syndromes from IPS, where routine MR imaging does not show structural abnormalities. In MSA, hypointensity of the basal ganglia on T2weighted images is frequently observed, sometimes in combination with a lateral putaminal hyperintensity. ${ }^{1,2}$ However at higher magnetic field strengths (3T and above), this pattern can also be found in healthy elderly subjects and its utility is, therefore, debatable. ${ }^{3}$ In the recent consensus criteria, those features have not been incorporated, though brain atrophy defined by MR imaging has been included as an additional clinical definition criterion for possible MSA. ${ }^{4}$ Midbrain atrophy is a hallmark for PSP and is commonly used as an MR

Received February 8, 2011; accepted after revision April 1, 2011

From the Department of Clinical Neurophysiology (N.K.F., P.M.P., S.S., C.G.B., W.P.), MR-Research in Neurology and Psychiatry (G.H., P.D.), and Department of Neuroradiology (M.K.), Georg-August University Göttingen, Göttingen, Germany; and Paracelsus Elena Klinik (N.K.F., J.E., C.T.), Specialized Movement Disorders Hospital, Kassel, Germany.

Please address correspondence to: Niels Focke, MD, Department of Clinical Neurophysiology, Georg-August University Göttingen, Robert-Koch-Str 40, 37099 Göttingen, Germany; e-mail: nfocke@uni-goettingen.de

Indicates article with supplemental on-line tables

Indicates article with supplemental on-line figure.

http://dx.doi.org/10.3174/ajnr.A2865 imaging criterion, ${ }^{5}$ though it is not included in the current definition criteria of PSP. ${ }^{6}$

All these radiologic signs are based on standard MR imaging acquisitions and qualitative visual inspection of the images. On the other hand, MR imaging offers the unique ability to gain quantitative parameter estimates reflecting the microstructure of the brain in vivo. Quantitative MRI is increasingly used to investigate normal aging and neurodegeneration. So far, DTI, magnetization transfer expressed with the MTR, and $\mathrm{T} 2$ and $\mathrm{T} 2{ }^{*}$ relaxometry have been used $^{7}$ to scrutinize the anatomic involvement and dynamics of the pathologic processes in patients with IPS and/or APS, but they are also used in clinical decision-making. To unravel which quantitative method (DTI, magnetization transfer imaging, T2/T2* relaxometry) is best suited for clinical purposes and to understand more about the differences in the underlying neurodegenerative processes, we conducted a head-to-head quantitative MR imaging study including healthy elderly controls and patients with IPS, PSP, and MSA-type Parkinson.

\section{Materials and Methods}

\section{Subjects}

Thirty-one patients (12 with IPS, 10 with MSA-type Parkinson, and 9 with PSP) and 13 healthy elderly subjects were enrolled in the study after written informed consent was obtained. Patients were recruited consecutively from in- and outpatients who fulfilled the following criteria: MSA diagnosed according to clinical consensus criteria, ${ }^{4}$ PSP according to the criteria of Litvan et al, ${ }^{6}$ and IPS according to UK 


\begin{tabular}{lccc}
\hline \multicolumn{4}{l}{ Demographic details of controls and patient groups } \\
\hline & No. & Age (mean) & Sex (female/male) \\
\hline Controls & 13 & $67.6 \pm 10.5$ & $8: 5$ \\
IPS & 12 & $66.3 \pm 7.8$ & $2: 10$ \\
MSA-P & 10 & $62.5 \pm 8.5$ & $5: 5$ \\
PSP & 9 & $67.0 \pm 4.3$ & $2: 7$ \\
Total & 44 & $66.0 \pm 8.3$ & $17: 27$ \\
\hline
\end{tabular}

Note:-MSA-P indicates multiple systems atrophy-type Parkinson.

Brain Bank Criteria ${ }^{8}$ without MR imaging contraindications or concomitant brain disease. Demographic details are shown in the Table. The control group's mean age was $67.6 \pm 10.5$ years ( 8 women), the IPS group's mean age was $66.3 \pm 7.8$ years ( 2 women), the MSA-type Parkinson group's mean age was $62.5 \pm 8.5$ years ( 5 women), and the PSP group's mean age was $67.0 \pm 4.3$ years ( 2 women). The overall mean age was $66.0 \pm 8.3$ years. There was no statistically significant difference ( 2 -tailed $P<.05$ ) between the groups concerning age (independent-samples $t$ test) or sex (Mann-Whitney $U$ test).

All patients with APS (MSA-type Parkinson and PSP) were recruited from the Paracelsus Elena Klinik, Kassel, Germany, a specialized movement disorders hospital. Patients with IPS were recruited from the University Hospital Göttingen and the Paracelsus Elena Klinik, Kassel, Germany. Clinical details of the patients enrolled are given in On-line Table 1. All cases were re-reviewed in 2009 before the final analysis for proof of consistency. A control group of neurologically healthy elderly subjects was recruited from the general population in the Göttingen area by advertisements and from congregations and leisure groups. The study was approved by the University Hospital of Göttingen ethics committee.

\section{Imaging Protocol}

MR imaging examinations were performed on a 3T whole-body MR imaging system (Magnetom Trio; Siemens, Erlangen, Germany) by using the body coil for transmission and an 8-channel head coil for signal-intensity reception (Invivo, Gainesville, Florida). The protocol comprised structural MR imaging scans with 1-mm isotropic resolution (T1-weighted: MPRAGE, TE $=3.26 \mathrm{~ms}, \mathrm{TR}=2250 \mathrm{~ms}$, TI $=900$ ms; T2-weighted: TSE with variable refocusing flip angles along a train of 198 echoes, $\mathrm{TE}_{\text {eff }}=427 \mathrm{~ms}$, TR $\left.=2900 \mathrm{~ms}\right)$.

A spoiled gradient-echo 3D sequence (FLASH) with 10 in-phase echoes $(\mathrm{TE}=4.92,9.84,14.8,19.7,24.6,29.5,34.4,39.4,44.3$ and 49.2 $\mathrm{ms}, \mathrm{TR}=54 \mathrm{~ms}, \alpha=16^{\circ}$ ) and a $1.7-\mathrm{mm}$ isotropic resolution was used for $\mathrm{R} 2^{*}$-mapping $\left(\mathrm{R} 2^{*}=1 / \mathrm{T} 2^{*}\right)$. TE range and resolution were chosen similar to those in established protocols at 3T. ${ }^{9}$ For R2-mapping, a multi-spin-echo sequence was used with a $1.7-\mathrm{mm}$ in-plane resolution and a 3.4-mm section thickness. Only even echoes were evaluated (at $\mathrm{TE}=30,60,90,120$, and $150 \mathrm{~ms}$, $\mathrm{TR}=4500 \mathrm{~ms}$ ) because these are not contaminated by stimulated echo signals. Magnetization transfer and R1-mapping was performed by using a nonselective 3D FLASH acquisitions with an isotropic resolution of 1.25 $\mathrm{mm}$ as previously described. ${ }^{10}$ In addition to the MT-weighted dataset (12.8-ms Gaussian pulse of $540^{\circ}$ flip angle applied $2.2-\mathrm{kHz}$ offresonance; TR $\left.=25 \mathrm{~ms}, \alpha=5^{\circ}\right), 2$ reference datasets were acquired with proton-attenuation weighting and T1-weighting (TR $=11 \mathrm{~ms}$, $\alpha=5^{\circ}$ and $\left.15^{\circ}\right)$. DTI was measured with a spin-echo-prepared $(\mathrm{TE}=$ $50 \mathrm{~ms}, 24$ directions of diffusion-weighting with $b=950 \mathrm{~s} / \mathrm{mm}^{2}$ and 5 nonweighted references) single-shot STEAM sequence of TE $=$ $7 \mathrm{~ms}, \mathrm{TR}=15.44$ seconds accommodating 38 contiguous sections of 2.2-mm isotropic resolution. ${ }^{11,12}$ Flip angle mapping was based on 2 single STEAM acquisitions of 3.5-mm isotropic resolution ( $\mathrm{TE}=6$ $\mathrm{ms} ; \mathrm{TR}=22.4 \mathrm{~ms}$, accommodating 54 sections $).{ }^{13}$

\section{Image Processing}

The DICOM images were transferred to a Linux-based image server and converted to 3D Neuroimaging Informatics Technology Initiative format by using "MRIconvert" (http://lcni.uoregon.edu/ $\sim$ jolinda/MRIConvert/). Further image processing was done with the Functional MR Imaging of the Brain Software Library (FSL 4.1 of the Centre for Functional Magnetic Resonance of the Brain, University of Oxford, United Kingdom; http://www.fmrib.ox.ac.uk/fsl/). For DTI, the FDT toolkit was used. FA and MD maps were calculated after correction for eddy currents by using "dtifit." Maps of R2 and R2* relaxation rates were calculated by logarithmic regression with the respective TEs. The dual-angle FLASH references yielded maps of signal-intensity amplitude and R1 (after correction for flip angle inhomogeneities). In turn, these maps were used to calculate MT saturation maps from the magnetization transfer-weighted FLASH, thus accounting for $\mathrm{T} 1$ and flip angle effects. ${ }^{10,14}$ This method also provided MTR maps. All structural volumes and quantitative parameter maps were coregistered by using the "FLIRT" tool by the following procedure: First, the T1-weighted MPRAGE volumes were aligned to the Montreal Neurologic Institute 152 template by a rigid body transformation $(6 d f)$ to create an individual T1-weighted template of 1 -mm resolution. Registration of the T2-weighted TSE volume provided a T2-weighted template for the measurements based on T2weighted images (DTI, R2, and R2*). All quantitative maps were then registered to individual space by concatenating the transformation matrixes from the space of the individual maps to the 3D T1-weighted images. Thus, every map was resampled only once to avoid progressive smoothing by consecutive registration steps.

\section{Quality Control}

All images and the resulting maps were visually inspected and excluded from further analysis when they showed significant artifacts (eg, due to motion) or had technical problems. In total, R2*-mapping failed in 2 control subjects, 1 patient with IPS and 1 with MSA. R2mapping failed in 1 control subject, 1 patient with IPS, and 1 with MSA. DTI was unavailable in 1 patient with IPS and 1 with PSP. Magnetization transfer/T1 mapping was not possible in 1 patient with IPS (details in On-line Table 1). Also, images were screened visually for concomitant lesions; no patient or control showed any specific or more unspecific lesions than would be expected for this elderly cohort.

\section{Region-of-Interest Analysis}

$3 \mathrm{D}$ regions of interest were placed in the $\mathrm{PU}, \mathrm{PA}, \mathrm{CN}$, and $\mathrm{SN}$, individually on both sides (Figs 1 and 2). Regions of interest were manually drawn by using "MRIcron" (http://www.sph.sc.edu/comd/ rorden/mricron/) by a single operator blinded to the diagnosis (P.M.P.) and cross-checked on both the T1-weighted images and MT parameter maps. The latter was particularly important for the SN, which is only poorly discernable on standard T1-weighted images but was clearly visible as a hypointensity on the magnetization transfer maps (compare On-line Fig 1). Only distinct gray matter voxels were included, and regions of interest were visually inspected to ensure absence of any gross artifacts. The mean values of each quantitative map were then determined in turn for each region of interest by using "fslstats." 

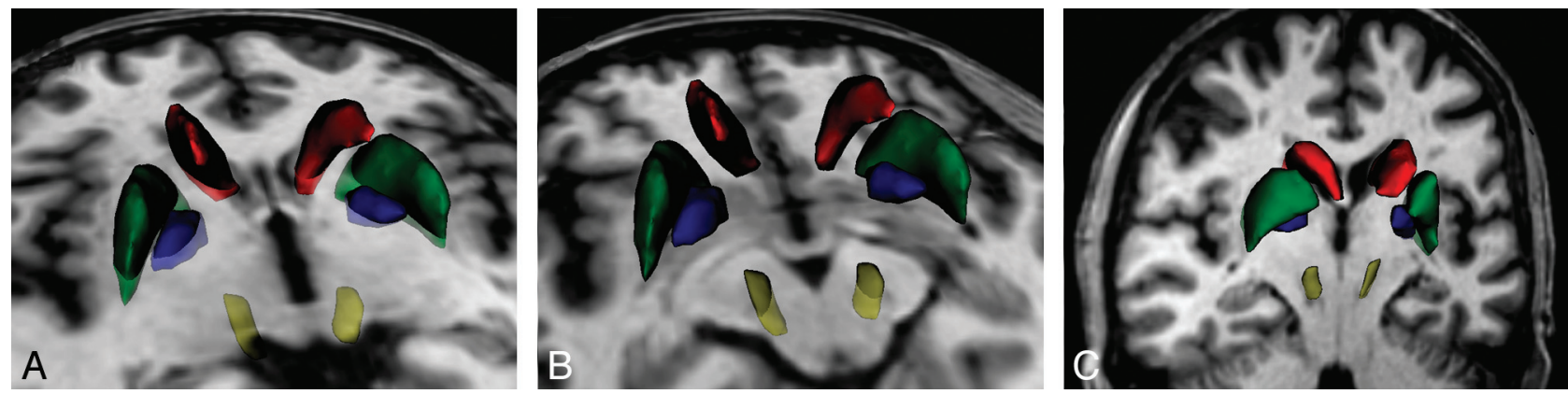

Fig 1. 3D rendering of the regions of interest in a control subject: CN (red), PU (green), PA (blue), and SN (yellow). Regions of interest overlaid on the 3D T1-weighted image. A, Axial cut plane at the level of the striatum. $B$, Axial cut plane through the mesencephalon. $C$, Coronal oblique cut plane along the main axis of the $\mathrm{SN}$ region of interest.
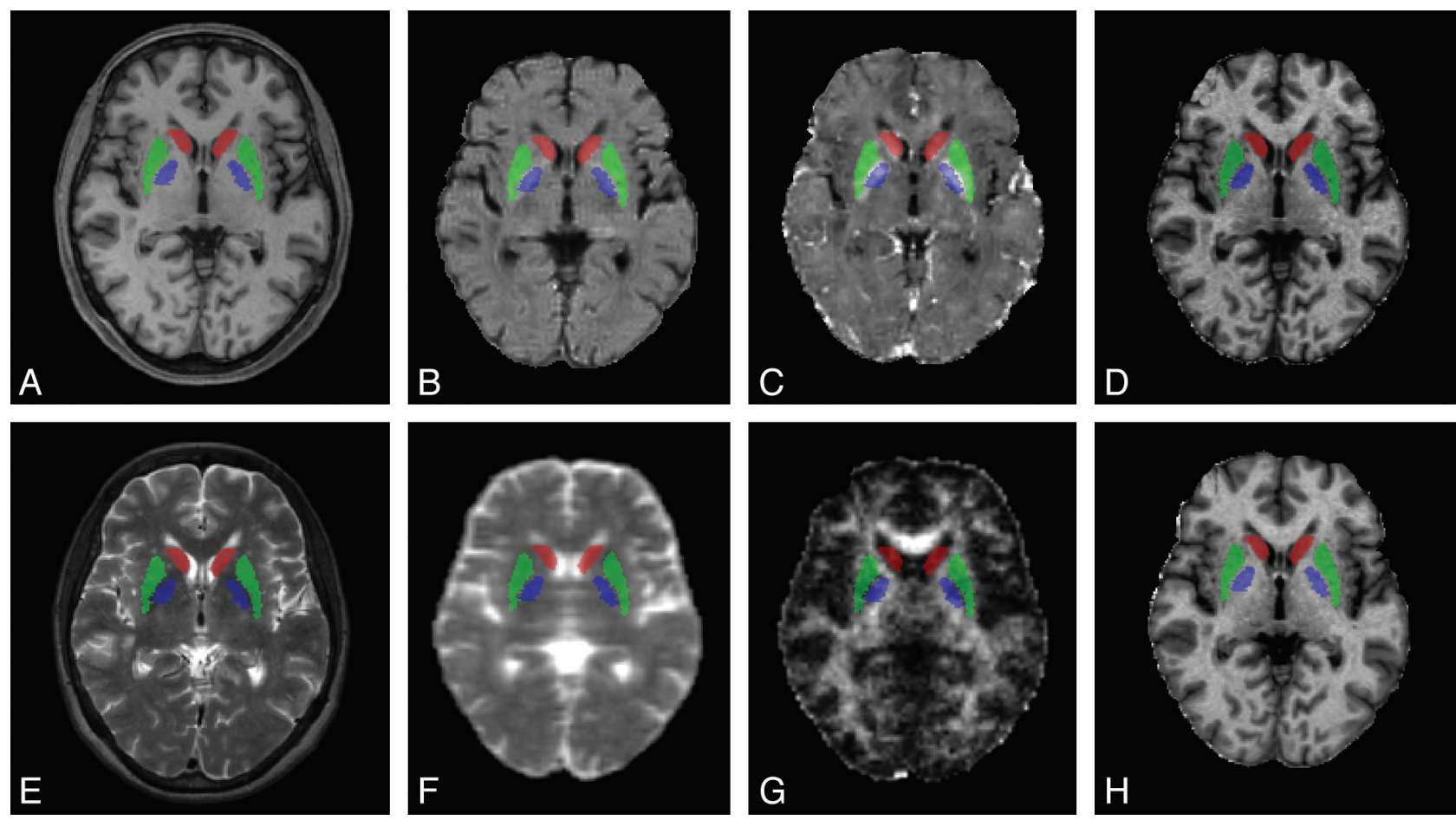

Fig 2. Regions of interest overlaid on the quantitative maps: CN (red), PU (green), and PA (blue). Regions of interest overlaid on axial sections of a 3D T1-weighted image (A), an R2 map $(B)$, an $\mathrm{R}^{*}$ map $(C)$, a magnetization transfer map $(D)$, a 3D T2-weighted image $(E)$, MD $(F)$, FA $(G)$, and R1 map $(H)$.

\section{Statistics}

Statistical analysis was done with PASW, Version 18 (SPSS, Chicago, Illinois). Means and SDs were calculated for each quantitative map and each group (controls, IPS, MSA-type Parkinson, and PSP), and a post hoc ANOVA (corrected for multiple comparisons with a Bonferroni error correction) was performed to analyze significant differences between the groups. Also ROC curves were plotted and the AUC was calculated to assess which method gave the best group separation. To account for the multiple comparison problem in the ROC analysis, we considered as significant only findings passing an uncorrected significance threshold of $P<.05 /$ number of group comparisons $=P<.0083$.

\section{Results}

Mean parameter values and SDs for each analyzed region of interest and patient group are given in On-line Table 2. $P$ values of the 1-way ANOVA comparing the groups are shown in On-line Table 3.

IPS Versus Controls. When comparing IPS patients with controls, we detected an increased magnetization transfer in the left $\mathrm{CN}$. No other significant differences were detected in any of the quantitative maps.

MSA-type Parkinson versus Controls. Between the MSAtype Parkinson and the control group, there were significant increases of $2^{*}$ in the PU bilaterally and the right PA. Ninetyfive percent confidence intervals for the right side $\mathrm{R} 2^{\star}$ measurements are shown in Fig 3. No significant differences were detected in the MD/FA, magnetization transfer, MTR, R1, or R2 maps.

MSA-type Parkinson versus IPS. When comparing patients with MSA-P and IPS, again we could detect significant $\mathrm{R} 2 *$ increases in the PU bilaterally and the right PA. Also decreased MTR values were found in the PU bilaterally and the left SN. No significant differences were found in the magnetization transfer, R1, R2, and MD/FA maps.

PSP versus Controls. Between patients with PSP and controls, significant MD increases were found in the PA and SN bilaterally. Also, in the $\mathrm{CN}$, a significant FA increase was detected on the right side; an R1 increase, bilaterally; and a mag- 


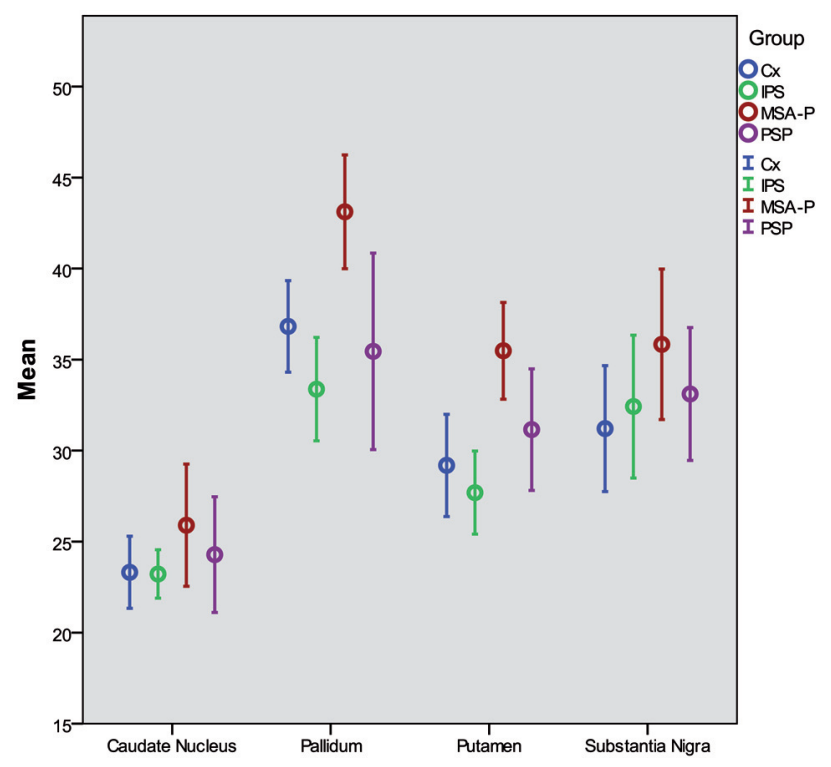

Fig 3. Group differences of $\mathrm{R} 2{ }^{*}$ measurements. Error bars ( $95 \%$ confidence intervals) of $\mathrm{R} 2^{*}$ measurements (right side only) are shown clustered by diagnosis group.

netization transfer increase, on the left side. No significant differences could be found in the R2, R2*, or MTR measurements in any of the regions of interest.

PSP versus IPS. When we compared patients with PSP and IPS, a similar pattern with significant MD increases was found in the right PA and SN (a trend was found on the left). In the MTR measurements, reduced values were detected in the right PA (a trend was again found on the left). No significant changes were found in the FA, R2, R2*, magnetization transfer, and R1 maps.

MSA-type Parkinson versus PSP. Finally when we compared patients with MSA-type Parkinson and PSP, R2 values were higher for the MSA-type Parkinson group in the PA bilaterally and $\mathrm{R} 2{ }^{*}$ values in the right $\mathrm{PA}$. MD values were higher in the PSP group in the SN bilaterally. No significant differences were found in the other maps.

\section{ROC Analysis}

Detailed results of the ROC analysis (AUC and $P$ values) are shown in On-line Table 3.

IPS versus Controls. When we compared patients with IPS and controls, no significant prediction (exceeding the strict $P$ value threshold) was possible with any of the applied methods. At trend level, we found an MD increase in the left SN, an MTR increase in both CNs, and a magnetization transfer increase in the left $\mathrm{CN}$.

MSA-type Parkinson versus Controls. In the comparison of patients with MSA-type Parkinson and controls, $\mathrm{R} 2^{\star}$ had a significant predictive value in the PU bilaterally and the right PA. Also, there was a significant predictive power for $\mathrm{R} 1$ in the right $\mathrm{SN}$.

MSA-type Parkinson versus IPS. When we contrasted patients with MSA-type Parkinson and IPS, R2* increases again showed a strong predictive power in the PU bilaterally and the right PA. Lower MTR values significantly predicted MSAtype Parkinson in the right PU (a trend was found contralaterally) and the left SN. Magnetization transfer, R2, R1, and
FA/MD could not differentiate the groups significantly better than chance in any region.

PSP versus Controls. In the comparison of patients with PSP and controls, we found a significant predictive power for $\mathrm{MD}$ in the left PA (a trend was found contralaterally) and in the SN bilaterally. With FA, a significant prediction was possible in the right $\mathrm{CN}$.

PSP versus IPS. In the comparison of patients with IPS and PSP, MD again showed some predictive power in the SN but did not reach the strict significance level.

MSA-P versus PSP. Finally when we compared the atypical Parkinson syndromes with each other, higher R2* values predicted MSA-type Parkinson in the right PA and higher MD values (at trend level) in the SN predicted PSP.

\section{Discussion}

When we compared patients with IPS with those with MSAtype Parkinson and healthy controls, our major finding was a significant predictive power for $\mathrm{R} 2^{\star}$-mapping in the PU. The other quantitative contrasts were less informative. For PSP, some predictive value was achieved with DTI in the SN.

\section{Quantitative MR Imaging in MSA-P}

The clinically most relevant finding resulted from $\mathrm{R} 2^{*}$ measurements between patients with IPS and MSA-type Parkinson, with a good predictive power $(A U C \leq 0.96)$ in the $P U$ and partially in the PA. With, for example, the right putaminal region of interest, a theoretic sensitivity (with specificity set to $100 \%$ ) of $77.8 \%$ could be achieved. When we aimed for $100 \%$ sensitivity, the resulting specificity would be $72.7 \%$. This is superior to what has been found in previous, qualitative MR imaging studies, ${ }^{1}$ but given the few cases, ours has to be interpreted with caution. Although signal-intensity decreases of the basal ganglia based on visual inspection of $\mathrm{T} 2^{*}$-weighted images have been previously described in MSA and (to a lesser extent) PSP, ${ }^{1,2,15}$ a quantitative approach (eg, by R2* measurement) allows objectively defining a normal range and, thus, increasing the confidence in a pathologic finding.

The high sensitivity of $\mathrm{R} 2{ }^{\star}$ to iron at higher magnetic field strengths can pose problems in standard $\mathrm{T}^{\star}{ }^{\star}$-weighted MR imaging. ${ }^{3}$ This can be turned into an advantage by using quantitative R2* measurements, which have been shown to be feasible up to magnetic field strengths of $7 \mathrm{~T} .{ }^{16}$ Most interesting, R2 mapping of patients with MSA-type Parkinson did show higher values in the basal ganglia as well, but the predictive power from these only reached a trend-level significance. The main difference between $\mathrm{R} 2$ and $\mathrm{R} 2{ }^{*}$ is the increased sensitivity to local field inhomogeneities as induced by the presence of iron. An increase in R2, and especially R2*, can, therefore, be regarded as a marker for iron accumulation, and several studies have provided neuropathologic evidence for this view. ${ }^{16-18}$ Our results indicate that the higher iron sensitivity of R2* is a key factor in detecting patients with MSA-type Parkinson. The other parameters in our study were clearly less useful in differentiating patients with MSA-type Parkinson from those with IPS or controls. DTI showed no significant predictive power in patients with MSA-type Parkinson, which is in contrast to previous reports ${ }^{19,20}$ and was evidently inferior to what could be achieved with $\mathrm{R} 2^{\star}$-mapping. This may be partly due to the DTI technique used in our study. To avoid image distor- 
tions and make region-of-interest analysis more spatially comparable, we used a STEAM-based DTI, which does not show susceptibility-related distortions but has an inherently lower signal intensity-to-noise ratio compared with conventional echo-planar imaging-based DTI.

Concerning the magnetization transfer-based imaging, we found that the traditional MTR showed reduced values in the PU (changes were stronger on the right side) and allowed statistically significant predictive power. This is in keeping with a previous study. ${ }^{21}$ The predictive power of this was, however, worse than that using $\mathrm{R} 2{ }^{\star}$-mapping. In addition, the MTR does not solely represent the magnetization transfer phenomenon but is also influenced by R1 and flip angle inhomogeneities. $^{22}$ We used a more specific concept for MT that corrects the steady-state for underlying changes of R1 and flip angle ${ }^{10}$ and thus improves the delineation of iron-containing deep brain structures. $^{23}$ The absence of significant differences by using this method indicates that the observed MTR differences may, in fact, be caused by other effects than magnetization transfer. In view of our results, it may be useful to combine R1-, R2*-, magnetization transfer-, and proton-attentuationmapping in a time-efficient high-resolution protocol. ${ }^{24}$

\section{Quantitative MR Imaging in PSP}

The pattern of quantitative MR imaging changes in PSP was different from that in the MSA-type Parkinson group. The most striking feature was an increase of MD in the SN compared with controls and (to a lesser extent) with patients with IPS and MSA-type Parkinson. MD values were also higher in the PA compared with both controls and patients with IPS. Previous studies have reported increased apparent diffusion coefficients in the basal ganglia regions, particularly in the PA/ $\mathrm{PU}$, and, recently, in the superior cerebellar peduncle. ${ }^{7,25,26}$ Although we could confirm the changes in the PA, a better separation was achieved by using the $\mathrm{SN}$ regions of interest; in comparison with MSA, the magnitude of differences and, thus, the predictive power was lower. In PSP, a profound volume loss in the brain stem is a typical feature and has been confirmed by volumetric MR imaging studies. ${ }^{27,28} \mathrm{It}$, therefore, is possible that DTI alterations are not confined to the SN region but could involve larger areas of the mesencephalon/ brain stem. This is, however, difficult to confirm in a regionof-interest-based study and should be assessed (eg, by a voxelbased approach), preferably in a larger cohort.

\section{Quantitative MR Imaging to Separate MSA-type Parkinson from PSP}

MSA and PSP are distinct pathologic entities; the first one accounts for a synucleinopathy, whereas the latter is assigned to tauopathies. There is, however, considerable overlap of the clinical and MR imaging phenotypes in both syndromes, sometimes revealing the correct diagnosis only after postmortem examination. ${ }^{29}$ Similarly, in our study, there was no good discriminator between the 2 atypical Parkinson syndromes. We observed some differences in the R2 (PA), R2*(PA and $\mathrm{PU}), \mathrm{R} 1(\mathrm{SN})$, and MD (PA, SN) measures, but most did not reach the strict $P$ value threshold in the ROC analysis. The clinical utility of these minor differences is, therefore, questionable.

\section{Quantitative MR Imaging in IPS}

Although the differentiation of patients with IPS from controls can mostly be done clinically, MR imaging can provide insights into the neurobiology of the disease. While routine clinical MR imaging findings are supposed to be normal in IPS ${ }^{30}$ quantitative MR imaging revealed some abnormalities in the $\mathrm{SN}$, mostly with $\mathrm{R} 2^{*}$ or $\mathrm{R} 2^{\prime}\left(\mathrm{R} 2^{\star}-\mathrm{R} 2^{\prime}\right)$ mapping. ${ }^{31-33} \mathrm{~A}$ recent study has also described pathologic changes of the substantia nigra with high-resolution DTI in patients with de novo IPS by using a single-section manual region of interest. ${ }^{34}$ In our sample, a borderline MD increase in the left $\mathrm{SN}$ was also observed but did not survive error correction. This could be due to technical differences of the DTI method applied or the different strategy used to define the regions of interest (3D versus single-section with 3 subregions).

\section{Limitations}

The diagnosis in our study was based on clinical findings only; so far no neuropathologic information is available. However, a recent study comparing the clinical MSA classification with neuropathologic validation showed a high positive predictive value of 95\% for "possible" and 100\% for "probable" cases. ${ }^{35}$ Also our study is relatively small. A lack of significant differences in any of the methods applied can, therefore, not exclude relevant changes in these paradigms in larger samples or differently selected subgroups. Because patients were selected to fulfill the diagnostic criteria for APS, additional matching (for age and sex) was not feasible. However, there was no statistically significant difference $(P>.05)$ between the groups for both potentially confounding parameters. Moreover, operator bias cannot be fully eliminated with manual region-ofinterest analysis. We tried to minimize this by using a single operator for the region-of-interest placement and automated image registration methods.

\section{Conclusions}

More studies with larger numbers are needed to further establish the value of quantitative MR imaging for the differentiation of patients with APS, ideally on a prospective basis with neuropathologic confirmation. From our data, R2*-mapping seems to be the most promising candidate for such an endeavor for patients with MSA, and DTI for patients with PSP. Multi-gradient-echo (with log-regression or monoexponential fitting to determine R2*) and DTI sequences are available on most clinical MR imaging systems. Our findings can, thus, have direct clinical implications.

Disclosures: Walter Paulus, Research Support (including provision of equipment or materials): DFG, BMBF; Speaker Bureau: Glaxo-Smith-Kline, UCB Pharma, Details: Educational tasks; Consultant: Desitin, Pfizer, EBS Technologies, Details: Consulting boards; Other Financial Interests: Danish Agency for Science, Deutsches Stiftungszentrum. Claudia Trenkwalder, Research Support (including provision of equipment or materials). TEVA Pharma Parkinson Study (DeNoPa); Speaker Bureau: Boehringer Ingelheim, Solvay, GlaxoSmith-Kline, UCB Schwarz Pharama, Novartis, Vifor Pharma, Axxonis Pharma; Consultant: Boehringer Ingelheim, UCB, Novartis, Axxonis Pharma.

\section{References}

1. von Lewinski F, Werner C, Jorn T, et al. T2*-weighted MRI in diagnosis of multiple system atrophy: a practical approach for clinicians. J Neurol 2007;254:1184-88

2. Kraft E, Schwarz J, Trenkwalder C, et al. The combination of hypointense and hyperintense signal changes on $\mathrm{T} 2$-weighted magnetic resonance imaging 
sequences: a specific marker of multiple system atrophy? Arch Neurol 1999;56:225-28

3. Lee WH, Lee CC, Shyu WC, et al. Hyperintense putaminal rim sign is not a hallmark of multiple system atrophy at 3T. AJNR Am J Neuroradiol 2005;26:2238-42

4. Gilman S, Wenning GK, Low PA, et al. Second consensus statement on the diagnosis of multiple system atrophy. Neurology 2008;71:670-76

5. Schrag A, Good CD, Miszkiel K, et al. Differentiation of atypical parkinsonian syndromes with routine MRI. Neurology 2000;54:697

6. Litvan I, Agid Y, Calne D, et al. Clinical research criteria for the diagnosis of progressive supranuclear palsy (Steele-Richardson-Olszewski syndrome): report of the NINDS-SPSP international workshop. Neurology 1996;47:1-9

7. Hotter A, Esterhammer R, Schocke MFH, et al. Potential of advanced MR imaging techniques in the differential diagnosis of parkinsonism. Mov Disord 2009;24:S711-20

8. Hughes AJ, Daniel SE, Kilford L, et al. Accuracy of clinical diagnosis of idiopathic Parkinson's disease: a clinico-pathological study of 100 cases. J Neurol Neurosurg Psychiatry 1992;55:181-84

9. Peran P, Hagberg G, Luccichenti G, et al. Voxel-based analysis of R2* maps in the healthy human brain. J Magn Reson Imaging 2007;26:1413-20

10. Helms G, Dathe H, Kallenberg K, et al. High-resolution maps of magnetization transfer with inherent correction for RF inhomogeneity and T1 relaxation obtained from 3D FLASH MRI. Magn Reson Med 2008;60:1396-407

11. Finsterbusch J, Frahm J. Half-Fourier single-shot STEAM MRI. Magn Reson Med 2002;47:611-15

12. Rieseberg S, Merboldt KD, Kuntzel M, et al. Diffusion tensor imaging using partial Fourier STEAM MRI with projection onto convex subsets reconstruction. Magn Reson Med 2005;54:486-90

13. Helms G, Finsterbusch J, Weiskopf N, et al. Rapid radiofrequency field mapping in vivo using single-shot STEAM MRI. Magn Reson Med 2008;60:739-43

14. Helms G, Dathe H, Dechent P. Quantitative FLASH MRI at 3T using a rational approximation of the Ernst equation. Magn Reson Med 2008;59:667-72

15. Kraft E, Trenkwalder C, Auer DP. T2*-weighted MRI differentiates multiple system atrophy from Parkinson's disease. Neurology 2002;59:1265-67

16. Yao B, Li TQ, Gelderen P, et al. Susceptibility contrast in high field MRI of human brain as a function of tissue iron content. Neuroimage 2009;44: 1259-66

17. Hardy PA, Gash D, Yokel R, et al. Correlation of R2 with total iron concentration in the brains of rhesus monkeys. J Magn Reson Imaging 2005;21:118-27

18. Matsusue E, Fujii S, Kanasaki Y, et al. Putaminal lesion in multiple system atrophy: postmortem MR-pathological correlations. Neuroradiology 2008;50: 559-67

19. Schocke MF, Seppi K, Esterhammer R, et al. Trace of diffusion tensor differentiates the Parkinson variant of multiple system atrophy and Parkinson's disease. Neuroimage 2004;21:1443-51

20. Seppi K, Schocke MFH, Esterhammer R, et al. Diffusion-weighted imaging discriminates progressive supranuclear palsy from PD, but not from the parkinson variant of multiple system atrophy. Neurology 2003;60:922-27

21. Naka H, Imon $Y$, Ohshita $T$, et al. Magnetization transfer measurements of brain structures in patients with multiple system atrophy. Neuroimage 2002;17:1572-78

22. Helms G, Dathe H, Dechent P. Modeling the influence of TR and excitation flip angle on the magnetization transfer ratio (MTR) in human brain obtained from 3D spoiled gradient echo MRI. Magn Reson Med 2010;64:177-85

23. Helms G, Draganski B, Frackowiak R, et al. Improved segmentation of deep brain grey matter structures using magnetization transfer (MT) parameter maps. Neuroimage 2009;47:194-98

24. Helms G, Dechent P. Increased SNR and reduced distortions by averaging multiple gradient echo signals in 3D FLASH imaging of the human brain at 3T. J Magn Reson Imaging 2009;29:198-204

25. Nicoletti G, Lodi R, Condino F, et al. Apparent diffusion coefficient measurements of the middle cerebellar peduncle differentiate the Parkinson variant of MSA from Parkinson's disease and progressive supranuclear palsy. Brain 2006;129:2679-87

26. Nicoletti G, Tonon C, Lodi R, et al. Apparent diffusion coefficient of the superior cerebellar peduncle differentiates progressive supranuclear palsy from Parkinson's disease. Mov Disord 2008;23:2370-76

27. Price S, Paviour D, Scahill R, et al. Voxel-based morphometry detects pattern of atrophy that help differentiate progressive supranuclear palsy and Parkinson's disease. Neuroimage 2004;23:663-69

28. Focke N, Helms G, Scheewe S, et al. Individual voxel-based subtype prediction can differentiate progressive supranuclear palsy from idiopathic Parkinson syndrome and healthy controls. Hum Brain Mapp 2011;32:1905-15

29. Litvan I, Goetz CG, Jankovic J, et al. What is the accuracy of the clinical diagnosis of multiple system atrophy? A clinicopathologic study. Arch Neurol 1997;54:937-44

30. Seppi K, Schocke MF. An update on conventional and advanced magnetic resonance imaging techniques in the differential diagnosis of neurodegenerative parkinsonism. Curr Opin Neurol 2005;18:370-75

31. Graham JM, Paley MNJ, Grunewald RA, et al. Brain iron deposition in Parkinson's disease imaged using the PRIME magnetic resonance sequence. Brain 2000;123:2423-31

32. Michaeli S, Oz G, Sorce DJ, et al. Assessment of brain iron and neuronal integrity in patients with Parkinson's disease using novel MRI contrasts. Mov Disord 2007;22:334-40

33. Gorell JM, Ordidge RJ, Brown GG, et al. Increased iron-related MRI contrast in the substantia nigra in Parkinson's disease. Neurology 1995;45:1138-43

34. Vaillancourt DE, Spraker MB, Prodoehl J, et al. High-resolution diffusion tensor imaging in the substantia nigra of de novo Parkinson disease. Neurology 2009;72:1378-84

35. Osaki Y, Ben-Shlomo Y, Lees AJ, et al. A validation exercise on the new consensus criteria for multiple system atrophy. Mov Disord 2009;24:2272-76 